\title{
High inductance planar transformers
}

\author{
O. Dezuari * , S.E. Gilbert, E. Belloy, M.A.M. Gijs \\ Institute of Microsystems, Swiss Federal Institute of Technology (EPFL), CH-1015 Lausanne, Switzerland
}

\begin{abstract}
We have developed a new printed circuit board (PCB) technology adapted for the fabrication of ultra-flat microtransformers. The two outer layers of the PCB stack comprise the electrical windings of the inductive devices, while the inner layer is made of a micro-patterned amorphous magnetic ribbon with extremely high relative magnetic permeability $\left(\mu_{\mathrm{r}} \approx 100,000\right)$. The electrical properties of the toroidal microtransformers have been studied as a function of frequency. Inductances were found to be as much as $30 \mu \mathrm{H}$ at $1 \mathrm{kHz}$, a relatively high value compared to microtransformers of similar size. Despite a relatively high leakage flux, the voltage transformation efficiency is measured at $89 \%$. The high performance of these devices is primarily due to the use of the high permeability magnetic core. (C) 2000 Elsevier Science S.A. All rights reserved.
\end{abstract}

Keywords: Planar transformers; PCB technology; High permeability material; Inductance; Vitrovac ${ }^{\circledR}$

\section{Introduction}

Miniaturisation of inductive devices for signal transformation, sensitive magnetic field detection or switch-mode power supply applications is an increasingly important subject today. Often these devices determine, to an important extent, the size of the complete application in which they are employed.

Recently, we have proposed a new technique for the realisation of inexpensive and flat transformer devices with relatively high inductance [1], compared with published results on micromachined transformers [2-6].

It may be clear that for an appreciable inductance value within a rather small volume, it is very important to have a core material within the inductor or transformer that has a very high relative magnetic permeability $\mu_{\mathrm{r}}$. Today, magnetic alloys with extremely high $\mu_{\mathrm{r}}$ values can be fabricated by splash-cooling the molten alloy into an amorphous state. One type of such material, which is commercially available, is the Vitrovac ${ }^{\circledR}$ family of magnetic alloys. For this work, a particular alloy, known under the trade name Vitrovac ${ }^{\circledR}$ 6025, (composition (Co,Fe, $\left.\mathrm{Mo})_{73}(\mathrm{Si}, \mathrm{B})_{27}\right)$, was chosen primarily because of its extremely high relative permeability $\left(\mu_{\mathrm{r}} \approx 100,000\right)$ [7]. This material is already employed as core material for three-dimensional magnetic amplifier chokes, where the transition

\footnotetext{
* Corresponding author
}

from a high inductance at low fields to a low inductance at saturation is used in a high speed on/off switch for power converter applications [8].

Our flat transformers are composed of three layers of which the outer layers carry the printed coil patterns and the inner layer is a Vitrovac ${ }^{\circledR} 6025$ high permeability ferromagnetic sheet core. Both magnetic metal and copper layers are patterned using standard lithographic techniques. In between the magnetic metal and copper layers there is a sheet of solid epoxy glue (Prepreg ${ }^{\circledR}$ typically $100 \mu \mathrm{m}$ ) for insulation and assembly. Such material is well known in the assembly of multilayer PCB structures. Connection between the outer copper patterns is realised by electroplating methods to complete the windings. These transformers are about $600 \mu \mathrm{m}$ thick, with lateral dimensions approximately $1 \mathrm{~cm}$ and inductance values in the $10 \mu \mathrm{H}$ range.

\section{Transformer design}

Fig. 1 shows a design diagram for a toroidal transformer. This diagram represents in transparency the two external layers and the internal one. The internal layer that represents the toroidal magnetic core is characterised by an outer radius $A(6.9 \mathrm{~mm})$ and an inner radius $B(3.9 \mathrm{~mm}$ or $5.3 \mathrm{~mm}$ ). One should note that in this figure, the primary windings are divided into two groups, which have been 


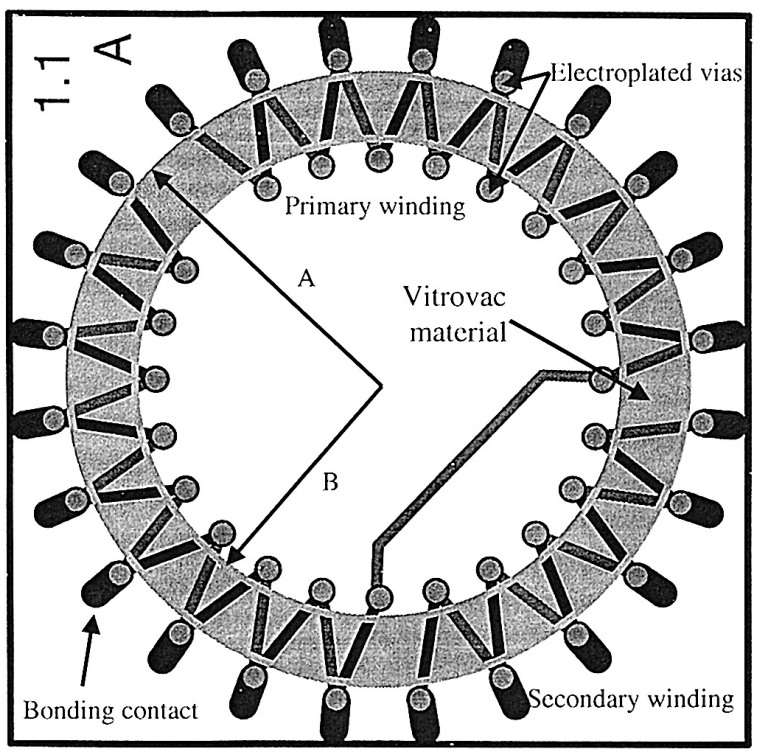

Fig. 1. Schematic design diagram of a toroidal $\mu$-transformer.

symmetrically positioned at both sides of the secondary windings. A typical ratio for the number of turns between the primary and secondary windings $N_{\text {prim }} / N_{\text {sec }}$ is $18 / 5$.

A photograph of the experimental realisation of a toroidal transformer is displayed in Fig. 2.

\section{Fabrication process}

A schematic diagram of the coil fabrication process is shown in Fig. 3. The proposed device fabrication is very similar to a conventional PCB process. These devices are based on two epoxy boards (100 $\mu \mathrm{m}$ thick) with copper (35 $\mu \mathrm{m}$ thick) laminated on one side, and by a simple epoxy board (100 $\mu \mathrm{m}$ thick) as support for the Vitrovac ${ }^{\circledR}$

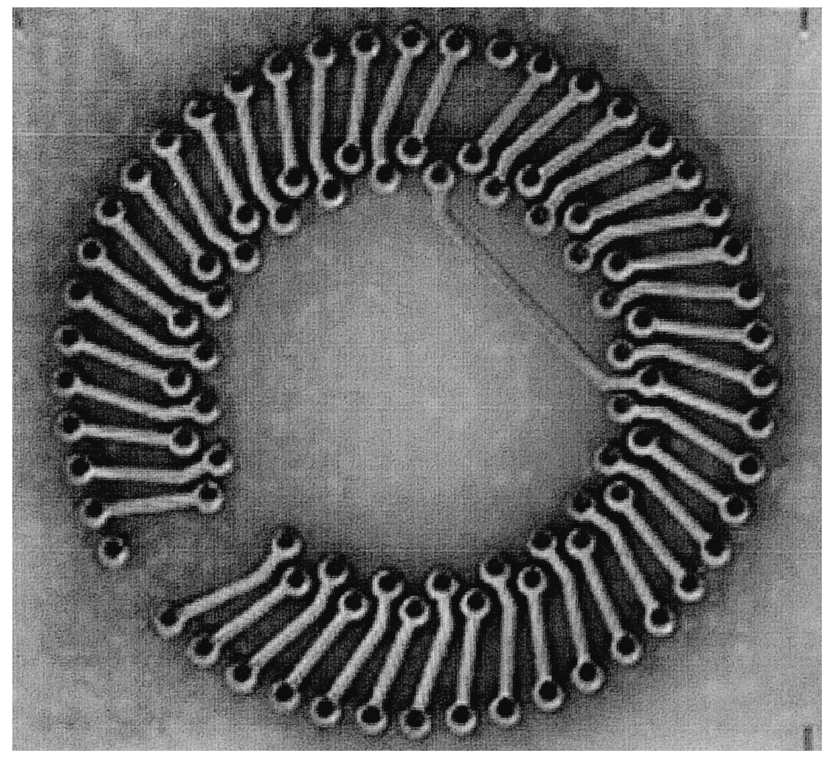

Fig. 2. Photograph of a finished toroidal $\mu$-transformer.

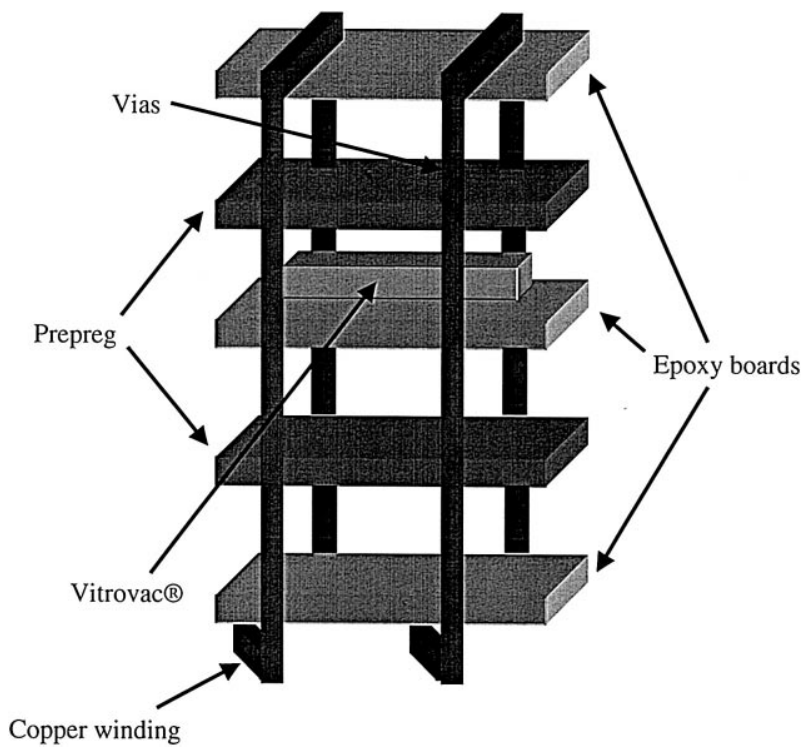

Fig. 3. Schematic diagram of the transformer micro-fabrication process.

magnetic foil. A liquid epoxy was used to laminate the magnetic foil on the inner epoxy board. Two $100 \mu \mathrm{m}$-thick sheets of prepreg solid epoxy were used for bonding the laminates into a stack of five layers.

The Vitrovac ${ }^{\circledR} 6025$ foil was patterned photolithographically to form the transformer cores by first laminating sheets of solid negative photoresist (Ordyl ${ }^{\circledR} 200$ [9]) onto degreased foils, and then processing according to manufacturer's specifications after pattern transfer. A wet chemical process was developed in-house to etch the magnetic foils, consisting of exposure of the resist-covered foils to a freshly prepared aqua regia type etching bath $\left(\mathrm{HNO}_{3}: \mathrm{HCl}: \mathrm{H}_{2} \mathrm{O}, 2: 1: 3\right)$ for several minutes at room temperature without agitation. The photoresist proved itself immune to the etchant, and exhibited excellent adhesion if the substrates were well cleaned.

To align the layers, a pinning system was used. The precision of the alignment holes define the maximum lateral precision available with this technology (currently about $100 \mu \mathrm{m}$ ). Bonding operations were carried out by hot pressing typically at $180^{\circ} \mathrm{C}$ and $20 \mathrm{~kg} / \mathrm{cm}^{2}$. During the bonding step, the epoxy sheets melt, whereby the viscous liquid spreads out and contacts all interior surfaces under the applied pressure. Via holes were drilled at appropriate positions for the interconnects, which were subsequently metallised using copper electroplating. Winding patterns were produced on the outer copper layers photolithographically, thereby completing the helical coil pattern which fully encircles the interior core.

\section{Results}

\subsection{Main inductance and resistance}

Typical frequency response characteristics of the primary main inductance, $L_{\mathrm{m}, \mathrm{p}}$, of toroidal transformers are 


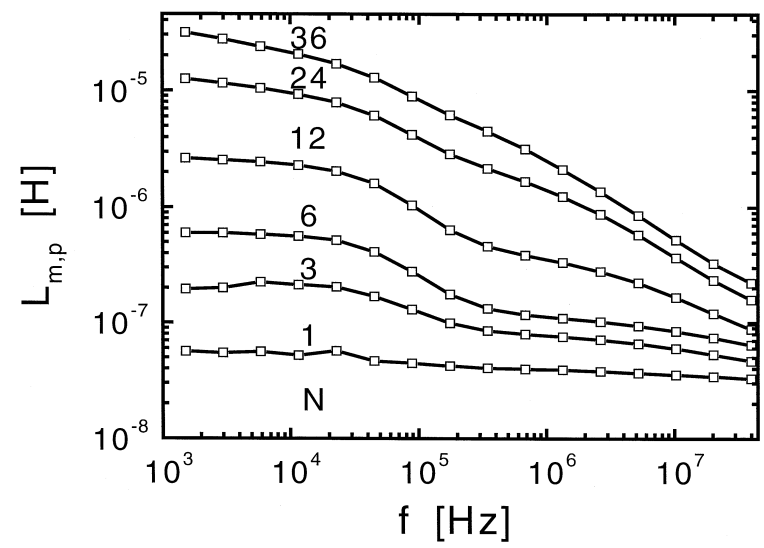

Fig. 4. Inductance versus frequency for toroidal microtransformers with varying number of turns $N$. The inner radius $B$ of the magnetic material of this transformer is $5.3 \mathrm{~mm}$.

shown in Fig. 4, for different number of windings $N$. These curves were obtained with an open secondary. The monotonic decrease of $L_{\mathrm{m}, \mathrm{p}}$ with frequency is in part due to the intrinsic behaviour of the magnetic material, which exhibits a similar dependence [8]. Fig. 4 clearly shows that $L_{\mathrm{m}, \mathrm{p}}$ increases with increasing $N$, an effect predicted to follow a parabolic power law $\left(N^{2}\right)$ for an ideal inductor. The power law dependence of the toroidal transformer is shown in Fig. 5. The deviations from $N^{2}$ behaviour at low winding number (and hence, low intrinsic $L_{\mathrm{m}, \mathrm{p}}$ ) may be due to spurious lead inductance and capacitive effects.

\subsection{Transformer gain measurements}

Fig. 6 is a typical gain $\left(V_{\text {sec }} / V_{\text {prim }}\right)$ plot for a toroidal microtransformer. The gain plot is a measure of the real voltage transformation ratio, and is indicative of the microtransformers' performance over a wide frequency range. In Fig. 6, the gain for a toroidal transformer in step-down configuration is plotted. This graph reveals that it has a flat response of $-12 \mathrm{~dB}$ starting at about $20 \mathrm{kHz}$ and extending to about $1 \mathrm{MHz}$. This decibel level corresponds to a

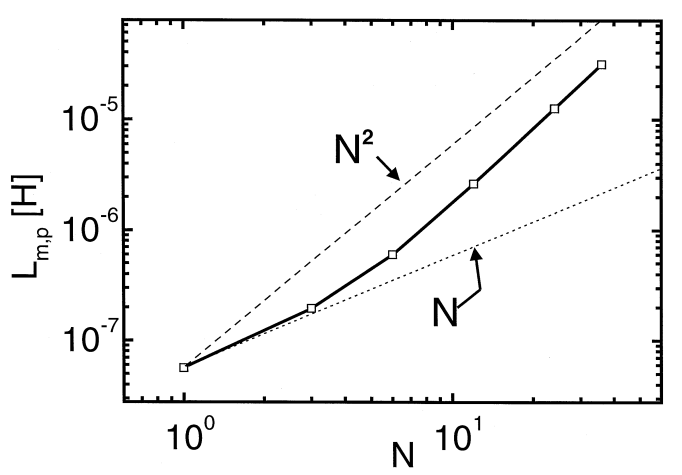

Fig. 5. Power-law dependence of primary inductance $L_{\mathrm{m}, \mathrm{p}}$ on number of windings $N$ for toroidal transformers. The dashed and dotted curves represent $N^{2}$ and $N$-like dependence respectively.

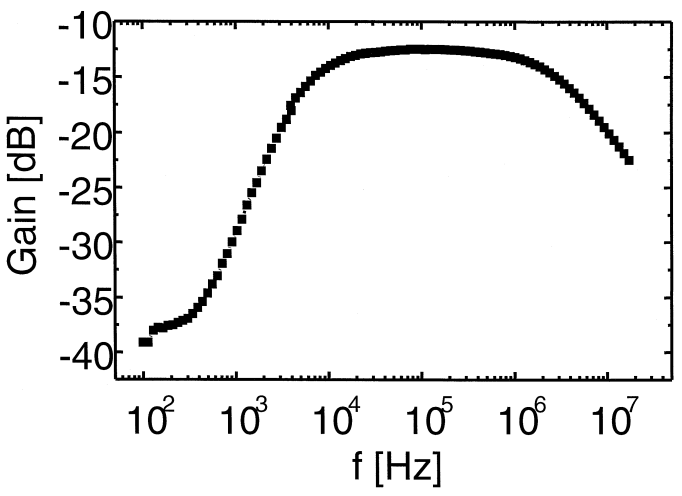

Fig. 6. Frequency dependence of transformation gain for a toroidal transformer.

voltage ratio of 0.25 . Taking into account the turns ratio of $18: 5$, ideally one would expect a voltage transformation ratio of 0.28 . Thus the measurement shows that this microtransformer has a $89 \%$ voltage transformation efficiency over almost two decades of frequency in the step-down configuration.

\section{Conclusions}

The data presented in this paper demonstrate that the proposed hybrid PCB-like technology is capable of producing planar microtransformers with performance characteristics that are equal to and even surpass those of previously reported devices of similar size. For the latter, more costly thin film fabrication techniques were employed in their fabrication. The frequency range for maximum efficiency has been shown to span a range of $20 \mathrm{kHz}$ to 1 MHz. This new hybrid PCB/magnetic metal foil technology permits the facile development of high inductance planar components. In comparison with existing planar inductor technologies, this new process virtually eliminates the need for time consuming and expensive thin film deposition techniques, while retaining compatibility with standard electronic packaging schemes. The use of industrially compatible processes based on inexpensive epoxy board substrate permits the reduction of costs and facilitates mass production at later stages.

\section{References}

[1] O. Dezuari, S.E. Gilbert, E. Belloy, M.A.M. Gijs, A new hybrid technology for planar microtransformer fabrication, submitted to Sensors and Actuators A, 1998

[2] J.Y. Park, L.K. Lagorce, M.G. Allen, Ferrite-based integrated planar inductors and transformers fabricated at low-temperature, IEEE Trans. Magn. 33 (1997) 3322-3324.

[3] B. Löchel, A. Maciossek, M. Rothe, W. Windbracke, Microcoils fabricated by UV depth lithography and galvanoplating, Sensors and Actuators A 54 (1996) 663-668.

[4] M. Mino, T. Yachi, A. Tago, K. Yanagisawa, K. Sakakibara, A new 
planar microtransformer for use in micro-switching converters, IEEE Trans. Magn. 28 (1992) 1969-1973.

[5] M. Mino, T. Yachi, A. Tago, K. Yanagisawa, K. Sakakibara, Planar microtransformer with monolithically integrated rectifier diodes for micro-switching converters, IEEE Trans. Magn. 32 (1996) 291-296.

[6] K. Yamasawa, K. Maruyama, I. Hirohama, P.P. Biringer, High frequency operation of a planar-type microtransformer and its applica- tion to multilayered switching regulators, IEEE Trans. Magn. 26 (1990) 1204-1209.

[7] Vitrovac ${ }^{\circledR}$ product literature, Vacuumschmelze, Hanau, Germany.

[8] R. Boll, Weichmagnetische Werkstoffe, (Vacuumschmelze, Hanau, Germany 1990).

[9] Ordyl ${ }^{\circledR}$ AF 200 product literature, ELGA RONAL, Milan, Italy. 